FACTA UNIVERSITATIS (NIŠ)

Ser. Math. Inform. Vol. 35, No 2 (2020), 311-320

https://doi.org/10.22190/FUMI2002311A

\title{
$f$-BIHARMONIC CURVES WITH TIMELIKE NORMAL VECTOR ON LORENTZIAN SPHERE
}

\author{
Bilal Eftal Acet
}

(C) 2020 by University of Niš, Serbia | Creative Commons Licence: CC BY-NC-ND

\begin{abstract}
In this paper, we study $f$-biharmonic curves as the critical points of the $f$-bienergy functional $E_{2}(\psi)=\int_{M} f\left|\tau(\psi)^{2}\right| \vartheta_{g}$, on a Lorentzian para-Sasakian manifold $M$. We give necessary and sufficient conditions for a curve such that has a timelike principal normal vector on lying a 4-dimensional conformally flat, quasiconformally flat and conformally symmetric Lorentzian para-Sasakian manifold to be an $f$-biharmonic curve. Moreover, we introduce proper $f$-biharmonic curves on the Lorentzian sphere $S_{1}^{4}$.

Keywords: $f$-biharmonic curves; $f$-bienergy functional; para-Sasakian manifold; Lorentzian sphere.
\end{abstract}

\section{Introduction}

Harmonic maps $\psi:(M, g) \rightarrow(N, h)$ between Riemannian manifolds are the critical points of the energy functional defined by

$$
E(\psi)=\frac{1}{2} \int_{\Omega}|d \psi|^{2} \vartheta_{g},
$$

for every compact domain $\Omega \subset M$. The Euler-Lagrange equation of the energy functional gives the harmonic equation defined by vanishing of

$$
\tau(\psi)=\operatorname{trace} \nabla d \psi,
$$

where $\tau(\psi)$ is called the tension field of the map $\psi$.

As a generalization of harmonic maps, biharmonic maps between Riemannian manifolds were introduced by J. Eells and J.H. Sampson [7]. Biharmonic maps

Received December 18, 2019; accepted April 03, 2020

2010 Mathematics Subject Classification. Primary 58E20; Secondary 53C25, 53C43 
between Riemannian manifolds $\psi:(M, g) \rightarrow(N, h)$ are the critical points of the bienergy functional

$$
E_{2}(\psi)=\frac{1}{2} \int_{\Omega}|\tau(\psi)|^{2} \vartheta_{g}
$$

for any compact domain $\Omega \subset M$.

In [3], G.Y. Jiang derived the first and the second variation formulas for the bienergy, showing that the Euler-Lagrange equation associated to $E_{2}$ is

$$
\begin{aligned}
\tau_{2}(\psi) & =-J^{\psi}(\tau(\psi)) \\
& =-\triangle \tau(\Psi)-\operatorname{trace}^{N}(d \psi, \tau(\psi)) d \psi,
\end{aligned}
$$

where $J^{\psi}$ is the Jacobi operator of $\psi$. The equation $\tau_{2}(\psi)=0$ is called biharmonic equation. Clearly, any harmonic maps is always a biharmonic map. A biharmonic map that is not harmonic is called a proper biharmonic map.

For some recent geometric study of biharmonic maps see [14, 17, 18, 19, 24] and the references therein. Also for some recent progress on biharmonic submanifolds see $[1,2,16,20,21]$ and for biharmonic conformal immersions and submersions see $[15,25,27]$.

The concept of $f$-biharmonic maps were initiated by W.J. Lu [23]. A smooth map $\psi:(M, g) \rightarrow(N, h)$ between Riemannian manifolds is called an $f$-biharmonic map if it is a critical point of the $f$-bienergy functional defined by

$$
E_{2, f}(\psi)=\frac{1}{2} \int_{\Omega} f|\tau(\psi)|^{2} \vartheta_{g},
$$

for every compact domain $\Omega \subset M$.

The Euler-Lagrange equation gives the $f$-biharmonic map equation [23]

$$
\begin{aligned}
\tau_{2, f} & =f \tau_{2}(\psi)+(\triangle f) \tau(\psi)+2 \nabla_{\text {gradf }}^{\psi} \tau(\psi) \\
& =0,
\end{aligned}
$$

where $\tau(\psi)$ and $\tau_{2}(\psi)$ are the tension and bitension fields of $\psi$, respectively. Therefore, we have the following relationship among these types of maps [26]:

$$
\text { Harmonic maps } \subset \text { Biharmonic maps } \subset f-\text { Biharmonic maps. }
$$

From now on we will call an $f$-biharmonic map, which is neither harmonic nor biharmonic, a proper $f$-biharmonic map (see also [28]).

The study of Lorentzian almost paracontact manifold was initiated by K. Matsumoto [9]. He also introduced the notion of Lorentzian para-Sasakian manifold. In [4], I. Mihai and R. Rosca defined the same notion independently and there after many authors [5, 11, 22] studied Lorentzian para-Sasakian manifolds.

Moreover, in [17] some geometric result for spacelike and timelike curves in a 4-dimensional conformally flat, quasi-conformally flat and conformally symmetric Lorentzian para-Sasakian manifold to be proper biharmonic were given. Motivated by this work, we introduced $f$-biharmonic curves on Lorentzian para-Sasakian manifold and Lorentzian sphere $S_{1}^{4}$. 


\section{Preliminaries}

\section{1. $\quad f$-Biharmonic Maps}

$f$-Biharmonic maps are critical points of the $f$-bienergy functional for maps $\psi:(M, g) \rightarrow(N, h)$ between Riemannian manifolds:

$$
E_{2, f}(\psi)=\frac{1}{2} \int_{\Omega} f|\tau(\psi)|^{2} \vartheta_{g},
$$

where $\Omega$ is a compact domain of $M$.

The following Theorem was proved in [23]:

Theorem 2.1. A map $\psi:(M, g) \rightarrow(N, h)$ between Riemannian manifolds is an $f$-biharmonic map if and only if

$$
\tau_{2, f}=f \tau_{2}(\psi)+(\triangle f) \tau(\psi)+2 \nabla_{\text {gradf }}^{\psi} \tau(\psi)=0,
$$

where $\tau(\psi)$ and $\tau_{2}(\psi)$ are the tension and bitension fields of $\psi$, respectively. $\tau_{2, f}(\psi)$ is called the $f$-bitension field of map $\psi$.

A special case of $f$-biharmonic maps is $f$-biharmonic curves. We have the following.

Lemma 2.1. [26] An arclength parametrized curve $\gamma:(a, b) \rightarrow\left(N^{m}, g\right)$ is an $f$-biharmonic curve with a function $f:(a, b) \rightarrow(0, \infty)$ if and only if

$$
f\left(\nabla_{\gamma^{\prime}}^{N} \nabla_{\gamma^{\prime}}^{N} \nabla_{\gamma^{\prime}}^{N} \gamma^{\prime}-R^{N}\left(\gamma^{\prime}, \nabla_{\gamma^{\prime}}^{N} \gamma^{\prime}\right) \gamma^{\prime}\right)+2 f^{\prime} \nabla_{\gamma^{\prime}}^{N} \nabla_{\gamma^{\prime}}^{N} \gamma^{\prime}+f^{\prime \prime} \nabla_{\gamma^{\prime}}^{N} \gamma^{\prime}=0 .
$$

\subsection{Lorentzian almost paracontact manifolds}

Let $M$ be an $n$-dimensional differentiable manifold with a Lorentzian metric $g$, i.e., $g$ is a smooth symmetric tensor field of type $(0,2)$ such that at every point $p \in M$, the tensor

$$
g_{p}: T_{p} M \times T_{p} M \rightarrow R,
$$

is a non-degenerate inner product of signature $(-,+,+, \ldots,+)$, where $T_{p} M$ is the tangent space of $M$ at the point $p$. Then $(M, g)$ is called a Lorentzian manifold. A non-zero vector $X_{p} \in T_{p} M$ can be spacelike, null or timelike, if it satisfies $g_{p}\left(X_{p}, X_{p}\right)>0, g_{p}\left(X_{p}, X_{p}\right)=0$ or $g_{p}\left(X_{p}, X_{p}\right)<0$, respectively.

Let $M$ be an $n$-dimensional differentiable manifold equipped with a structure $(\varphi, \xi, \eta)$, where $\varphi$ is a $(1,1)$-tensor field, $\xi$ is a vector field, $\eta$ is a 1 -form on $M$ such that $[9]$

$$
\varphi^{2} X=X+\eta(X) \xi,
$$




$$
\eta(\xi)=-1 .
$$

The above equations imply that

$$
\eta \circ \varphi=0, \quad \varphi \xi=0, \quad \operatorname{rank}(\varphi)=n-1 .
$$

Then $M$ admits a Lorentzian metric $g$, such that

$$
g(\varphi X, \varphi Y)=g(X, Y)+\eta(X) \eta(Y),
$$

and $M$ is said to admit a Lorentzian almost paracontact structure $(\varphi, \xi, \eta, g)$. Then we get

$$
g(X, \xi)=\eta(X) .
$$

The manifold $M$ endowed with a Lorentzian almost paracontact structure $(\varphi, \xi, \eta, g)$ is called a Lorentzian almost paracontact manifold $[9,10]$. In equations $(2.4)$ and (2.5) if we replace $\xi$ by $-\xi$, we obtain an almost paracontact structure on $M$ defined by I. Sato [6].

A Lorentzian almost paracontact manifold equipped with the structure $(\varphi, \xi, \eta, g)$ is called a Lorentzian para-Sasakian manifold [9] if

$$
\left(\nabla_{X} \varphi\right) Y=g(X, Y) \xi+\eta(Y) X+2 \eta(X) \eta(Y) \xi .
$$

The conformal curvature tensor $C$ is given by

$$
\begin{aligned}
C(X, Y) W= & R(X, Y) W-\frac{1}{n-2}\left\{\begin{array}{c}
S(Y, W) X-S(X, W) Y \\
+g(Y, W) Q X-g(X, W) Q Y
\end{array}\right\} \\
& +\frac{r}{(n-1)(n-2)}\{g(Y, W) X-g(X, W) Y\},
\end{aligned}
$$

where $S(X, Y)=g(Q X, Y)$. The Lorentzian para-Sasakian manifold is called conformally flat if conformal curvature tensor vanishes i.e., $C=0$.

The quasi-conformal curvature tensor $\hat{C}$ is defined by

$$
\begin{aligned}
\hat{C}(X, Y) W= & a R(X, Y) W-b\left\{\begin{array}{c}
S(Y, W) X-S(X, W) Y \\
+g(Y, W) Q X-g(X, W) Q Y
\end{array}\right\} \\
& -\frac{r}{n}\left(\frac{a}{(n-1)}+2 b\right)\{g(Y, W) X-g(X, W) Y\},
\end{aligned}
$$

where $a, b$ constants such that $a b \neq 0$. Similarly the Lorentzian para-Sasakian manifold is called quasi-conformally flat if $\hat{C}=0$.

We know that a conformally flat and quasi-conformally flat Lorentzian paraSasakian manifold $M^{n}(n>3)$ is of constant curvature 1 and also a Lorentzian para-Sasakian manifold is locally isometric to a Lorentzian unit sphere if the relation $R(X, Y) \cdot C=0$ holds on $M$ [12]. For a conformally symmetric Riemannian manifold [13], we get $\nabla C=0$. Thus for a conformally symmetric space the relation $R(X, Y)$. 
$C=0$ satisfies. Hence a conformally symmetric Lorentzian para-Sasakian manifold is locally isometric to a Lorentzian unit sphere [12].

Therefore, for a conformally flat, quasi-conformally flat and conformally symmetric Lorentzian para-Sasakian manifold $M$, we have [12]

$$
R(X, Y) W=g(Y, W) X-g(X, W) Y,
$$

for any vector fields $X, Y, W \in T M$.

\section{3. $\quad f$-Biharmonic Curves in Lorentzian Para-Sasakian Manifolds}

For a Lorentzian para-Sasakian manifold $M$, an arbitrary curve $\gamma: I \rightarrow M$, $\gamma=\gamma(s)$ is called spacelike, timelike or lightlike (null), if all of its velocity vectors $\gamma^{\prime}(s)$ are spacelike, timelike or lightlike (null), respectively. In this section, we give some conditions for a curve having timelike normal vector on a 4-dimensional conformally flat, quasi-conformally flat and conformally symmetric Lorentzian paraSasakian manifold $M$ to be an $f$-biharmonic curve.

Theorem 3.1. Let $\gamma: I \rightarrow M$ be a curve parametrized by arclength and $M$ be a 4-dimensional conformally flat, quasi-conformally flat and conformally symmetric Lorentzian para-Sasakian manifold. Asuume that $\left\{T, N, B_{1}, B_{2}\right\}$ be an orthonormal Frenet frame field along $\gamma$ such that principal normal vector $N$ is timelike. Then $\gamma$ is a proper $f$-biharmonic curve if and only if one of the following cases happens:

i) The first curvature $\kappa_{1}$ of the $\gamma$ solves the following ordinary differential equation,

$$
3\left(\kappa_{1}^{\prime}\right)^{2}-2 \kappa_{1} \kappa_{1}^{\prime \prime}=4 \kappa_{1}^{4}-4 \kappa_{1}^{2}
$$

with $f=t_{1} \kappa_{1}^{-\frac{3}{2}}$ and $\kappa_{2}=0$.

ii) The first curvature $\kappa_{1}$ of the $\gamma$ solves the following ordinary differential equation,

$$
3\left(\kappa_{1}^{\prime}\right)^{2}-2 \kappa_{1} \kappa_{1}^{\prime \prime}=4 \kappa_{1}^{4}+4 \kappa_{1}^{4} t_{3}^{2}-4 \kappa_{1}^{2}
$$

with $f=t_{1} \kappa_{1}^{-\frac{3}{2}}, \kappa_{2} \neq 0, \kappa_{3}=0, \frac{\kappa_{2}}{\kappa_{1}}=t_{3}$.

Proof. Let $\gamma$ be a curve parametrized by arclength on lying a 4-dimensional conformally flat, quasi-conformally flat and conformally symmetric Lorentzian paraSasakian manifold $M$ and let $\left\{T, N, B_{1}, B_{2}\right\}$ be an orthonormal Frenet frame field along $\gamma$ such that principal normal vector $N$ is timelike.

In this case for this curve, the Frenet frame equations are given by [8]

$$
\left[\begin{array}{c}
\nabla_{T} T \\
\nabla_{T} N \\
\nabla_{T} B_{1} \\
\nabla_{T} B_{2}
\end{array}\right]=\left[\begin{array}{cccc}
0 & \kappa_{1} & 0 & 0 \\
\kappa_{1} & 0 & \kappa_{2} & 0 \\
0 & \kappa_{2} & 0 & \kappa_{3} \\
0 & 0 & -\kappa_{3} & 0
\end{array}\right]\left[\begin{array}{c}
T \\
N \\
B_{1} \\
B_{2}
\end{array}\right]
$$


where $T, N, B_{1}, B_{2}$ are mutually orthogonal vectors and $\kappa_{1}, \kappa_{2}$ and $\kappa_{3}$ are respectively the first, the second and the third curvature of the $\gamma$.

In view of the Frenet formulas given in (3.3) and equation (2.8), we obtain

$$
\begin{gathered}
\nabla_{T} T=\kappa_{1} N, \\
\nabla_{T} \nabla_{T} T=\kappa_{1}^{2} T+\kappa_{1}^{\prime} N+\kappa_{1} \kappa_{2} B_{1}, \\
\nabla_{T} \nabla_{T} \nabla_{T} T= \\
\left(3 \kappa_{1} \kappa_{1}^{\prime}\right) T+\left(\kappa_{1}^{\prime \prime}+\kappa_{1}^{3}+\kappa_{1} \kappa_{2}^{2}\right) N \\
+\left(2 \kappa_{1}^{\prime} \kappa_{2}+\kappa_{1} \kappa_{2}^{\prime}\right) B_{1}+\left(\kappa_{1} \kappa_{2} \kappa_{3}\right) B_{2},
\end{gathered}
$$

and

$$
R\left(T, \nabla_{T} T\right) T=-\kappa_{1} N,
$$

where $\kappa_{1}, \kappa_{2}$ and $\kappa_{3}$ are the first, the second and the third curvature of the $\gamma$, respectively.

Considering Theorem 2.1 and equation (2.3), we get

$$
\begin{aligned}
\tau_{2, f}= & f\left[\begin{array}{c}
\left(3 \kappa_{1} \kappa_{1}^{\prime}\right) T+\left(\kappa_{1}^{\prime \prime}+\kappa_{1}^{3}+\kappa_{1} \kappa_{2}^{2}+\kappa_{1} N\right) \\
+\left(2 \kappa_{1}^{\prime} \kappa_{2}+\kappa_{1} \kappa_{2}^{\prime}\right) B_{1}+\left(\kappa_{1} \kappa_{2} \kappa_{3}\right) B_{2}
\end{array}\right] \\
& +2 f^{\prime}\left[\kappa_{1}^{2} T+\kappa_{1}^{\prime} N+\kappa_{1} \kappa_{2} B_{1}\right]+f^{\prime \prime}\left[\kappa_{1} N\right] \\
= & 0 .
\end{aligned}
$$

Comparing the coefficients of above equation, we obtain that $\gamma$ is an $f$-biharmonic curve if and only if

$$
\begin{gathered}
3 \kappa_{1} \kappa_{1}^{\prime}+2 \kappa_{1}^{2} \frac{f^{\prime}}{f}=0 \\
\kappa_{1}^{\prime \prime}+\kappa_{1}^{3}+\kappa_{1} \kappa_{2}^{2}+\kappa_{1}+2 \kappa_{1}^{\prime} \frac{f^{\prime}}{f}+\kappa_{1} \frac{f^{\prime \prime}}{f}=0 \\
2 \kappa_{1}^{\prime} \kappa_{2}+\kappa_{1} \kappa_{2}^{\prime}+2 \kappa_{1} \kappa_{2} \frac{f^{\prime}}{f}=0 \\
\kappa_{1} \kappa_{2} \kappa_{3}=0 .
\end{gathered}
$$

Let $\kappa_{1}$ be a non zero constant. Then from (3.4) we get $f$ is constant. So $\gamma$ is biharmonic. Let $\kappa_{2}$ be a non zero constant. From (3.4) and (3.6) one can easily see that $f$ is constant and $\gamma$ is biharmonic. 
By using (3.4) - (3.7), if $\kappa_{2}=0$, then $f$-biharmonic curve equation reduces to

$$
\begin{gathered}
3 \kappa_{1} \kappa_{1}^{\prime}+2 \kappa_{1}^{2} \frac{f^{\prime}}{f}=0, \\
\kappa_{1}^{\prime \prime}+\kappa_{1}^{3}+\kappa_{1}+2 \kappa_{1}^{\prime} \frac{f^{\prime}}{f}+\kappa_{1} \frac{f^{\prime \prime}}{f}=0 .
\end{gathered}
$$

Integrating the equation (3.8) we get $f=t_{1} \kappa_{1}^{-\frac{3}{2}}$ and using this result in (3.9), we arrive at $(i)$.

Otherwise, by use of (3.4) - (3.7), if $\kappa_{1} \neq$ constant and $\kappa_{2} \neq$ constant $f$-biharmonic curve the equation is equivalent to

$$
\begin{gathered}
f^{2} \kappa_{1}^{3}=t_{1}^{2}, \\
\left(f \kappa_{1}\right)^{\prime \prime}=-f \kappa_{1}\left(\kappa_{1}^{2}+\kappa_{2}^{2}+1\right), \\
f^{2} \kappa_{1}^{2} \kappa_{2}=t_{2}, \\
\kappa_{3}=0 .
\end{gathered}
$$

In view of (3.10), we find $f=t_{1} \kappa_{1}^{-\frac{3}{2}}$ and using this result in (3.11), we get $\frac{\kappa_{2}}{\kappa_{1}}=t_{3}$. Finally substituting these equation in (3.11), we arrive at (ii).

Proposition 3.1. Let $M$ be a 4-dimensional conformally flat, quasi-conformally flat and conformally symmetric Lorentzian para-Sasakian manifold and $\gamma: I \rightarrow M$ be an $f$-biharmonic spacelike curve parametrized by arclength such that principal normal vector is timelike. If $\gamma$ has constant geodesic curvature then $\gamma$ is biharmonic.

\section{4. $\quad f$-Biharmonic Curves on Lorentzian Sphere $S_{1}^{4}$}

Suppose that $M$ is a 4-dimensional conformally flat, quasi-conformally flat and conformally symmetric Lorentzian para-Sasakian manifold. Since $M$ is locally isometric to a Lorentzian unit sphere $S_{1}^{4}$, we give some characterizations for $f$-biharmonic curves in $S_{1}^{4}$. The Lorentzian unit sphere of radius 1 can be seen as the hyperquadradic

$$
S_{1}^{4}=\left\{p \in \mathbb{R}_{1}^{5}:<p, p>=1\right\},
$$

in a Minkowski space $\mathbb{R}_{1}^{5}$ with the metric

$$
<,>:-d x_{1}^{2}+d x_{2}^{2}+d x_{3}^{2}+d x_{4}^{2}+d x_{5}^{2} .
$$


Let $\gamma: I \rightarrow S_{1}^{4}$ be a curve parametrized by arclength. For an arbitrary vector field $X$ along $\gamma$, we have

$$
\nabla_{T} X=X^{\prime}+<T, X>\gamma
$$

where $\nabla$ is covariant derivative along $\gamma$ in $S_{1}^{4}$.

Since $S_{1}^{4}$ is a Lorentzian space form of the scalar curvature 1, we have

$$
R(X, Y) W=<Y, W>X-<X, W>Y,
$$

for all vector fields $X, Y, W$ in the tangent bundle of $S_{1}^{4}$, where $R$ is the curvature tensor of $S_{1}^{4}$.

Now, we give the following:

Proposition 4.1. Let $\gamma: I \rightarrow S_{1}^{4}$ be a non-geodesic $f$-biharmonic curve parametrized by arclength and $\left\{T, N, B_{1}, B_{2}\right\}$ be a Frenet frame along $\gamma$ such that

$$
g(T, T)=g\left(B_{1}, B_{1}\right)=g\left(B_{2}, B_{2}\right)=1, g(N, N)=-1 .
$$

Then, we have

$$
\gamma^{(4)}-\left(\frac{\kappa_{1}^{\prime \prime}}{\kappa_{1}}+2 \frac{\kappa_{1}^{\prime}}{\kappa_{1}} \frac{f^{\prime}}{f}+\frac{f^{\prime \prime}}{f}\right) \gamma^{\prime \prime}-\left(\kappa_{1}^{2}+\frac{\kappa_{1}^{\prime \prime}}{\kappa_{1}}+2 \frac{\kappa_{1}^{\prime}}{\kappa_{1}} \frac{f^{\prime}}{f}+\frac{f^{\prime \prime}}{f}+1\right) \gamma=0 .
$$

Proof. Using (3.5) and taking the covariant derivative of the second equation in (3.3), we get

$$
\begin{aligned}
\nabla_{T}^{2} N & =\nabla_{T}\left(\kappa_{1} T+\kappa_{2} B_{1}\right) \\
& =\kappa_{1} \nabla_{T} T+\kappa_{2} \nabla_{T} B_{1} \\
& =\left(\kappa_{1}^{2}+\kappa_{2}^{2}\right) N+\kappa_{2} \kappa_{3} B_{2}
\end{aligned}
$$

Using (3.5) in (4.3), we have

$$
\nabla_{T}^{2} N=-\left(\frac{\kappa_{1}^{\prime \prime}}{\kappa_{1}}+2 \frac{\kappa_{1}^{\prime}}{\kappa_{1}} \frac{f^{\prime}}{f}+\frac{f^{\prime \prime}}{f}+1\right) N
$$

On the other hand from (4.1), we arrive at

$$
\begin{aligned}
\nabla_{T}^{2} N & =\nabla_{T}\left(N^{\prime}+<T, N>\gamma\right) \\
& =N^{\prime \prime}+<T, N^{\prime}>\gamma \\
& =N^{\prime \prime}+<T, \nabla_{T} N-<N, T>\gamma>\gamma \\
& =N^{\prime \prime}+<T, \kappa_{1} T+\kappa_{2} B_{1}>\gamma \\
& =N^{\prime \prime}+\kappa_{1} \gamma .
\end{aligned}
$$

From (4.3) and (4.4), we obtain

$$
\left(\frac{\kappa_{1}^{\prime \prime}}{\kappa_{1}}+2 \frac{\kappa_{1}^{\prime}}{\kappa_{1}} \frac{f^{\prime}}{f}+\frac{f^{\prime \prime}}{f}+1\right) N=N^{\prime \prime}+\kappa_{1} \gamma
$$


Also in view of (4.1), we have

$$
\nabla_{T} T=T^{\prime}+<T, T>\gamma=\gamma^{\prime \prime}+\gamma
$$

which yields

$$
N=\frac{1}{\kappa_{1}}\left(\gamma^{\prime \prime}+\gamma\right) .
$$

By use of (4.5) and (4.4), we obtain (4.2).

\section{REF E R E N C E S}

1. A. Balmus, S. Montaldo and C. Oniciuc: Classification results for biharmonic submanifolds in spheres. Israel J. Math. 168 (2008), 201-220.

2. B. Y. Chen and M. VArga: Biharmonic ideal hypersurfaces in Euclidean spaces. Diff. Geom. Appl. 31 (2013), 1-16.

3. G. Y. JiAnG : 2-harmonic isometric immersions between Riemannian manifolds. Chinese Ann. Math. Ser. A. 7 (1986), 130-144.

4. I. Mihai and R. Rosca: Lorentzian P-Sasakian manifold in Classical Analysis. World Sci. Publ. River Edge, NJ, 1991, pp.155-169.

5. I. Minai, A. A. Shaikh and U. C. De: On Lorentzian para-Sasakian manifold. Rendicontidel Seminario Matematico di Messina, Serie II., 1999.

6. I. SAтo: On a structure similar to the almost contact structure. Tensor N.S. 30 (1976), 219-224.

7. J. Eells and J. H. SAMPSON: Harmonic mapping of the Riemannian manifold. American J. Math. 86 (1964), 109-160.

8. J. Walrave: Curves and surfaces in Minkowski spaces. Doctoral Thesis, K. U. Leuven, Fac. of Science, 1995.

9. K. Matsumoto: On Lorentzian paracontact manifolds. Bull. Yamagota Univ. Natur. Sci. 12 (1989), 151-156.

10. K. Matsumoto, I. Minai and R. Rosca: $\xi$-null geodesic gradient vector fields on a Lorentzian para-Sasakian manifold. J. Korean Math. Soc. 32 (1995), 17-31.

11. M. M. TRIPATHI and U. C. DE: Lorentzian almost paracontact manifolds and their submanifolds. J. Korean Soc. Math. Educ. Ser. B Pure Appl. Math. 8 (2001), 101-125.

12. M. TARAFDAR and A. Bhattacharyya: On Lorentzian para-Sasakian manifolds. In Steps in Differential Geometry (Debrecen). Inst. Math. Infor. (2000), 343-348.

13. M. C. CHAKI and B. Gupta: On conformally symmetric spaces. Indian J. Math. 5 (1963), 113-122.

14. P. BAIRD and D. KAmissoko: On constructing biharmonic maps and metrics. Ann. Glob. Anal. Geom. 23 (2003), 65-75.

15. P. Baird, A. FARdoun and S. OuAkKas: Conformal and semi-conformal biharmonic maps. Ann. Glob. Anal. Geom. 34 (2008), 403-414. 
16. R. Caddeo, S. Montaldo and C. Oniciuc: Biharmonic submanifolds of $S^{3}$. Int. J. Math. 12 (2001), 867-876.

17. S. Keleş, S. YÜKSel Perktaş and E. Kiliç: Biharmonic Curves in LP-Sasakian Manifolds. Bull. Malays. Math. Sci. Soc. 33 (2010), 325-344.

18. S. Montaldo and C. Oniciuc: A short survey on biharmonic maps between Riemannian manifolds. Rev. Un. Mat. Argent. 47 (2006), 1-22.

19. S. YÜKSEL PERKTAŞ and E. KILIÇ: Biharmonic maps between doubly warped product manifolds. Balkan Journal of Geometry and Its Appl. 15 (2010), 159-170.

20. S. YÜKSEl PeRkTAş, E. KiliÇ and S. Keleş: Biharmonic hypersurfaces of Lorentzian para-Sasakian manifolds. An. Stiint. Univ. Al. I. Cuza Iasi, Tomul LVII, f.2 DOI: 10.2478/v10157-011-0034-z (2011).

21. J. I. InOguchi and T. SASAhara: Biharmonic hypersurfaces in Riemannian symmetric spaces I. Hiroshima Math. J. 46 (2016), 97-121.

22. S. YÜksel Perktaş, E. KiliÇ and S. Keleş: Hypersurfaces of Lorentzian paraSasakian manifolds. Math. Scand. 108 (2011), 5-21.

23. W. J. Lu and R. S. VARGA: On $f$-Biharmonic maps between Riemannian manifolds. arXiv:1305.5478, preprint, (2013).

24. Y. L. OU: Biharmonic hypersurfaces in Riemannian manifolds. Pacific J. Math. 248 (2010), 217-232.

25. Y. L. Ou: On conformal biharmonic immersions. Ann. Glob. Anal. Geom. 36 (2009), 133-142.

26. Y. L. Ou: On f-biharmonic maps and $f$-biharmonic submanifolds. Pacific J. Math. 271 (2014), 461-477.

27. Z. P. WANG and Y. L. OU: Biharmonic Riemannian submersions from 3-manifolds. Math Z. 269 (2011), 917-925.

28. B. E. Acet, S. Yüksel Perktaş and F. E. Erdogan: A study on f-biharmonic curves in S(1,4). AIP Conference Proceed. 1991 (2018).

Bilal Eftal Acet

Faculty of Arts and Science

Department of Mathematics

P. O. Box 02040

Adiyaman, Turkey

eacet@adiyaman. edu.tr 